\title{
Confidence, Visual Research, and the Aesthetic Function
}

\author{
Stan Ruecker \\ Assistant Professor \\ Humanities Computing Program \\ Department of English and Film Studies \\ University of Alberta \\ Stéfan Sinclair \\ Communication Studies \& Multimedia \\ McMaster University \\ Milena Radzikowska \\ Electronic Publishing \& Technical Communication \\ Mount Royal College
}

\section{Abstract}

The goal of this article is to identify and describe one of the primary functions of aesthetic quality in the design of computer interfaces and visualization tools. We suggest that researchers in library and information science, computing science, and humanities computing can derive advantages in visual research by acknowledging - through their efforts to advance aesthetic quality - that a significant function of aesthetics in this context is to inspire the user's confidence. This confidence typically serves to create a sense of trust in the interface or tool, and to increase its perceived usability. In turn, this increased trust may result in an increased willingness to engage with the interface, on the basis that it demonstrates an attention to detail that promises to reward increased engagement. In addition to confidence, the aesthetic may also contribute to a heightened degree of satisfaction with having spent time using or investigating the object. In the realm of interface design and visualization research, we propose that these components of the aesthetic function (derived through trust) have implications not only for the quality of interactions, but also for the results of the standard measures of performance and preference.

Keywords: aesthetics, interface design, visualization, human-computer interaction

\section{Introduction}

Since the professionalization of various humanities disciplines in the latter part of the nineteenth century, humanities scholars have been primarily occupied with the interpretation and analysis of existing cultural artifacts, such as texts. The expertise and artistry required to produce the material form of the objects are generally outside the scope of a humanities education, and are placed instead within the fine arts. However, since the rise of personal computing and graphical 
interfaces, many humanities scholars have been empowered to create the interface through which their materials can be studied: the proliferation of digital collections such as Perseus (www.perseus.tufts.edu) and Rossetti (www.rossettiarchive.org) bears witness to this phenomenon. The distinctions between author, critic, editor and publisher have blurred.

Significantly though, the knowledge and perspective of artists and designers have either largely been ignored by the digital humanities scholar, or else have contributed in a manner that has not been subject to direct analysis. Whether working with basic web interfaces to text archives, or with projects that combine text and images, or with dynamically interactive experimental prototypes, researchers have the opportunity to extend their understanding through including the study of interface aesthetics alongside more traditional measures of performance and preference. The significance of the visual is sufficiently evident in all of these cases that aesthetic factors become intrinsically woven into issues of functionality (Dillon 2001, Petersen et al 2004). In addition to the larger area of computer interfaces in general, the growing interest in the more specialized field of humanities visualization systems is another research area where the study of design issues is relevant (Bradley and Rockwell 1994). Research interests in graphic design and presentation find a new relevance and weight, not only as a contributing factor, but also as an area of study in their own right.

For instance, Udsen and Jørgensen (2005) provide a summary of recent studies in interface aesthetics and create a taxonomy of four approaches: the cultural, the functionalistic, the experience-based and the techno-futuristic. Ngo et al. (2002) offer a model of interface aesthetics consisting of fourteen distinct characteristics. Bertelsen and Pold (2004) suggest adapting the process of art criticism for use in criticizing interface aesthetics. Hallnäs and Redström (2002) propose the noun "expressional" as a counterbalance to the adjective "functional," with the former term suggesting the components that go into the continuing presence of the designed computational object in everyday life. Fishwick et al. $(2005,2006)$ discuss the complex effects of art on computing.

We address the issue of graphic design contributions to visualization research in the context of several interdisciplinary interface design research projects at the University of Alberta, McMaster University, and York University. These projects include Watching the Script, (http://digitalplaybook.humviz.org, see Ruecker 2005, 2006, and Sinclair 2006) the Mandala Browser (http://mandala.humviz.org/ Ruecker 2006), Nora (http://noraproject.org/) and the Delegate Browser (Ruecker 2006), although in this article we will refer mostly to Watching the Script. Our emphasis is on the functional differences between early and later prototypes, including an analysis of the role of aesthetics. Our position is outlined by Frascara (1997, p. 14), who says, "aesthetics is one of the functions of design." We contend that the aesthetic function is a composite that includes attracting viewers, holding their attention, and compelling their trust and respect. Design, in 
other words, is of utmost importance to the value and legitimacy of scholarly digital content.

Previous studies have shown a significant relationship between perceived aesthetic quality and perceived usability for a variety of cultures. In Japan, Kurosu and Kashimura (1995) found that apparent usability correlates more strongly to aesthetic aspects of the interface than to actual usability. Tractinsky (1997) replicated their results in Israel. Karvonen (2000) takes this line of reasoning further in her Scandinavian study of the relationship between trust and design, finding that people tended to rate web sites with a clearly professional design quality as being more trustworthy than more vernacular sites.

The connection between graphic design and academic research also has implications for the ongoing need for improved communication between the academic and non-academic worlds. Several strategies are required at different levels, including public information campaigns, academic contributions to popular media (a good example is Andrew Vande Moere's blog at http://infosthetics.com/), and a significant presence of the academic in the community (which corresponds well to recent initiatives by Canadian granting agencies such as SSHRC, 2005). In this context of the academic's role as public intellectual, one potential role that design has to play is in visually rewarding the reader of research results.

It is a simple matter to identify a great many examples of academic work presented to the academic community and to the public in ways that are visually rewarding. One need go no further, really, than the Discovery Channel. There are still barriers to be overcome, however, in part because the academic world has other priorities, including clarity, correctness, and completeness, and in part for reasons of disciplinary visual culture. Fishwick (2006), in discussing some sophisticated network node diagrams created in Maya, suggests that a history of minimalism in computing science may have set the aesthetic bar relatively low: "Educated with minimalist figures and text, computer scientists may be shocked to realize our representations for formal objects are not as constrained as originally thought (p. 19)."

It might even be argued that there is an anti-aesthetic subtext in certain research areas, which emerges fairly naturally from an academic emphasis on content (substance) over form. An underlying assumption is that too much attention to form can suggest a hidden "marketing" agenda. A compelling example is Edward Tufte's treatise, "The Cognitive Style of PowerPoint" (Tufte 2003). However, such facile dichotomies can easily misrepresent the symbiosis of form and content, a fundamental relationship that has been recognized and studied at least since the Greek rhetoricians. Moreover, Pujol (2001) points out that the visual qualities of professional design are one of the key signifiers by which we distinguish the individual voice from the institutional. If someone hand-letters a sign to advertise a garage sale, we understand the sale as an amateur activity. If that same 
person employs graphic design skills and produces a glossy poster, we may interpret the same event, at least until we arrive at the site, as a professional rather than amateur activity.

To summarize, attention to the graphic quality of the design, particularly in the areas of visualization and information design research, can contribute both to the results obtained from user study, and to the reception of those research results. Careful attention to the details of graphic presentation can have a significant impact on the perceived value of a digital collection, the function of a visualization system, the research results available from analysis of visualizations, and the dissemination of findings both within the academic community and for the larger public audience.

\section{The Aesthetic Experience}

There is a considerable body of literature dealing with aesthetic theory, which ranges from the classical interest in discussing aesthetic factors, such as symmetry and balance, to postmodern questions about the cultural role of the aesthetic and the reasons for embracing instead the anti-aesthetic, particularly in fine art (Carroll 2001). From the perspective of perception and response, some theorists focus in particular on the aesthetic experience, which is generally understood to be a form of emotional reaction to a perceived object (Dufrenne 1973). A connection is commonly made between this emotional reaction and its purported function in civilizing us:

The value of beauty, then, is that along with human contact it enables us to break out of the otherwise impregnable spiritual isolation to which every one of us is born and to feel ourselves at home in the world. Beauty and friendship enable us to get outside ourselves and to live as we ought to live, in concord with the world we are part of, and to feel ourselves part of it (Pye 1978, p. 102).

Whether or not the perceived object needs to be a work of art per se is open to debate. Dufrenne (1973) takes it as a given for the purposes of his analysis, while Carroll (2001) challenges the assumption that there is any intrinsic connection, pointing out that many works of art are intended to promote other kinds of responses, and that in any case, the interpretive response is often as central as the aesthetic.

For some researchers, the aesthetic experience is a form of seduction. Khaslavsky and Shedroff (1999) discuss the seductive experience, breaking the process into three phases - enticement, relationship, and fulfillment - which translate into a range of requirements that must be met at different points in the relationship between the user and the object. 
Finally, a variety of theorists have attempted to operationalise the study of aesthetic experience, whether in its relation to art or to interfaces. Munro (1956), for instance, suggests an empirical approach using semantic differentials, similar to studies in other branches of sociology. Attempts have also been made to factor the aesthetic experience into its components. For example, Jennings (2000) defines the aesthetic experience as occurring when a person is consciously engaged in an activity that is immersive, pleasurable, unique, and personally rewarding.

\section{Design and Function}

Before we address the aesthetic function proper, it might be useful to describe in brief some of the various factors that are associated with the aesthetic, and which are influenced by it. The aesthetic, however, is not intrinsically reliant on these factors. That is, each factor can be successfully addressed in either an aesthetic or non-aesthetic way.

The first two factors are those typically discussed in the context of the design of public signage systems. The factors are whether the sign can be seen-whether it is discernible - and once it has been discerned, whether or not it is legible. In both these cases, the choices made by the designer will have an aesthetic dimension. There are instances of signage which are wonderfully discernible against their surrounds, and which are hallmarks for legibility once they have been discerned, for instance, by the anxious traveler. However, it is not essential that in order to meet these criteria, the signage also has to be beautiful. The aesthetic is possibly a component, but is neither necessary nor sufficient for the signage to perform its function.

A parallel instance occurs in the design of web pages, where the suggestion is often made by designers that part of their work consists of establishing meaningful arrangement of the visual elements, efficient arrangement of the menu items and other working parts, and a logical structure for navigation. Certainly these aspects of web design are within the purview of the designer. However, as in the case of signage, it is certainly possible to make a web page that features meaningful placement of items, clear navigation, and efficient arrangement of menus that is nonetheless not visually attractive. All one needs is the addition of an annoying and encumbering background pattern and a few distracting animated gifs, and the job is done.

Although the aesthetic can be a component of design decisions that also affect performance, the aesthetic and the functional have also often been disassociated. For instance, in many practical discussions, the two concepts are placed at difference poles, with the somewhat pejorative term "decorative" replacing the more positive connotations of the word "aesthetic." The aesthetic in these cases is almost invariably considered a kind of luxury, or indulgence, that can be dismissed until the real work is complete, and added on as a sort of icing 
or final polish to a project that might just as well do without the extra expense and complication.

Function and aesthetics are also occasionally linked, as in Frank Lloyd Wright's famous modernist formulation "form follows function." The principle here is that a particular kind of aesthetic_-one with a minimal or absent amount of ornamentation-can be seen as deriving from meeting performance requirements. The futuristic, streamlined look of the designs by Raymond Loewy or R. Buckminster Fuller, effectively illustrate this concept.

However, the various factors that involve the aesthetic can be logically identified, and the aesthetic component discussed separately. For example, it is reasonable to think of good typographic practices as involving an aesthetic component. Page layout is often described in terms of balance and harmony, both of which are aesthetic principles, but these attributes also contribute to the usefulness of the page as an object for sustained reading.

As in the earlier examples given for electronic media, however, it is also possible to have a printed page that serves very well as an object for sustained reading, which is not balanced or harmonious. One thinks, for instance, of pages created using a mechanical typewriter, where the conditions of production were not of the kind that could privilege considerations of experimental modification to line length or sophisticated adjustments of leading, and where font choice was minimal, if present as an option at all. Nonetheless, for two centuries people were able to read typewritten pages. So although it is possible to associate aesthetic value with page design, it is not reasonable to say that the aesthetic is intrinsic to the success of the page.

\section{The Aesthetic Function}

Our proposal is that the aesthetic function is not necessarily conscious, intentional, or telic. That is, it doesn't refer to the grosser acts of accomplishing a task. Instead, the aesthetic function is to provide the viewer, or in the case of visualization research the experimental participant, with implicit associations to the notions of quality and attention to detail. A primary component of the aesthetic function is therefore to inspire confidence, which results for visualization research in a number of advantages to the researcher, the participant in the research (or eventually the user of the system), and the larger community that receives and assesses the results.

This larger function of inspiring confidence can be divided into three subfunctions, related respectively to trust, willingness, and satisfaction. 


\section{Trust}

Trust is a factor in associations; it deals with the relationship between the user and the designer. In most cases, however, the designer won't be present when the user is interacting with the interface. It is therefore necessary for the designer to take measures to help instill a sense of trust through the mediation of the interface.

The opposite of trust is mistrust or fear. What do people using a visualization tool or computer interface have to fear, that trust would become an issue? They may fear that their time will be wasted, that they won't get their tasks completed properly or at all, and that the experience will be unpleasant. They may fear potentially nasty surprises. They may fear the lack of control associated with not clearly knowing what is going on. These are all reasonable fears, and they can be mitigated or even forestalled to the point that they never arise, through the provision of various aspects of the design intended to increase cognitive reassurance.

Cognitive reassurance can be provided in a variety of ways, but some of the factors that should be included are the ready availability of help and the provision of an environment that seems appropriate to accomplish the task. For example, a designer working with a vector drawing program expects to be able to readily find a tool that manipulates Bezier curves. Since this is only one of many such tools, drawing packages typically provide a tool palette that visually groups available tools. Someone working with a wordprocessor, however, would not expect a Bezier tool to be ready to hand - it isn't part of the appropriate environment. (See Maeda's The Laws of Simplicity (2006) for a discussion of negotiating between power and familiarity in an interface). Informed users want to see evidence of best practices in the interface. For example, a wide range of programs use files that can be saved, named, opened, and deleted. The best practice for the last twenty years has been to put these functions under a File menu, that sits near the top left on the screen. Users expect that File menu, and if it isn't there, they will wonder what is going on. They may not necessarily phrase their judgments in terms that explicitly involve the designer's competence, but might just respond subconsciously, or else think instead of their experience of carrying out the task (e.g. "that was unnecessarily hard") or in terms relating to a general perception of value (e.g. "that program sucks.") In some cases, where best practices change rapidly, it may happen that a particular design does not represent current best practice. However, even in these instances, if the design represents a previous best practice that the user can identify, there is a degree of reassurance possible.

A similar case can be made for the visual position of the design. Does it accommodate somebody's visual culture? Ideally, it should be positioned for the visual culture of the user; however, if there has clearly been attention paid to 
positioning it within some visual culture, that may be sufficient to indicate that the designer had some kind of user in mind.

Whether the cues are in the form of adoption of best practices or in the choice of visual position, or both, the goal should be to impress the user with every aspect of the tool. If everything about it looks considered, and it is clear that a reasonable allocation of resources has gone into its development, then the effect on the user will be to increase trust in the tool or its content, and - in cases where the producers of the resource are known - in the people responsible, which may include the designer, the researcher, and the developer. Since our context here is the design of experimental prototypes for academic research, the connection between designer and interface is stronger than is usually the case in online implementations. In the course of carrying out a usability research study, the designer, or at least the principal investigator, is usually identified for the participants by name. In the case of conference presentations, the designer is often in the room, delivering the paper, while in journal articles the designer is often listed as an author. The basic principle holds, however, even when the designers are anonymous or consolidated into a corporate provider, such as Microsoft, or a public organization, such as a university library.

A related set of cues have to do with the provision by the designer of visible indications that the interface or visualization system has been created in consultation with the relevant domain experts. These visible indications work in both directions: from the content expert to the designer and from the designer to the content expert. In this context, one indication of the professionalism of the researcher or developer who has the ultimate responsibility for the design can be the high level of professionalism apparent in the design.

\section{Willingness}

While trust implies a relationship between the designer and the user, willingness is a factor that reflects the user's internal state during the task. At a basic level, the question is whether or not the user is willing to take on the task at all, given the tools at hand. At a more sophisticated level, the question becomes to what extent the user is willing to persevere.

Perseverance is important throughout a task, but is particularly significant when something goes wrong. The user's willingness to engage in troubleshooting has to be predicated on the belief that the investment will be rewarded with success in overcoming the difficulty. During troubleshooting, the user puts in resources without receiving immediate benefit, based on confidence in a later benefit. Good aesthetic function demonstrates that the designer is trying to pack in rewards whenever rewards are possible.

At yet another level, there is the issue of morale, which is related in part to the influence of the environment over time. Morale is in some sense "willingness writ 
large," and the details that can encourage willingness can also support morale. As Pujol (2001) points out, it is important to consider the change in user experience with repeated exposure to the same design. Novelty and amusement can rapidly turn into repetition and irritation, as fashionable trends indicate in any number of fields, and interface design is no exception. However, it is also possible for the designer to create an environment where the attraction of the interface can persist and even grow through repeated exposure.

Micro Case-Study: Watching the Script

As an example, we present a prototype system for blocking and reading plays, called "Watching the Script" (Ruecker et al. 2004). The interface design has gone through three distinct stages, beginning with a white parallelogram, moving to a four-colour square, and ending with a very attractive full-colour combination of stage, playback controls, and large coloured dots that clearly indicate character positions (Figures 1a, 1b, and 1c).

Figures 1a, 1b, and 1c.
From top to bottom are
shown three stages in the
visual development of the
interface for the Watching
the Script project. This
sequence demonstrates the
increase in various kinds of
functionality, including the
aesthetic function.
Contributors to the project
include Stan Ruecker,
Stéfan Sinclair, Eric
Homich, Anthony Sapp, and
designers Sandra Gabriele
and Andrea Ruskin.

Figures $1 \mathrm{a}, 1 \mathrm{~b}$, and $1 \mathrm{c}$. From top to bottom are shown three stages in the visual development of the interface for the Watching the Script project. This sequence demonstrates the functionality, including the aesthetic function. Contributors to the project include Stan Ruecker, Stéfan Sinclair, Eric and Andrea Ruskin.

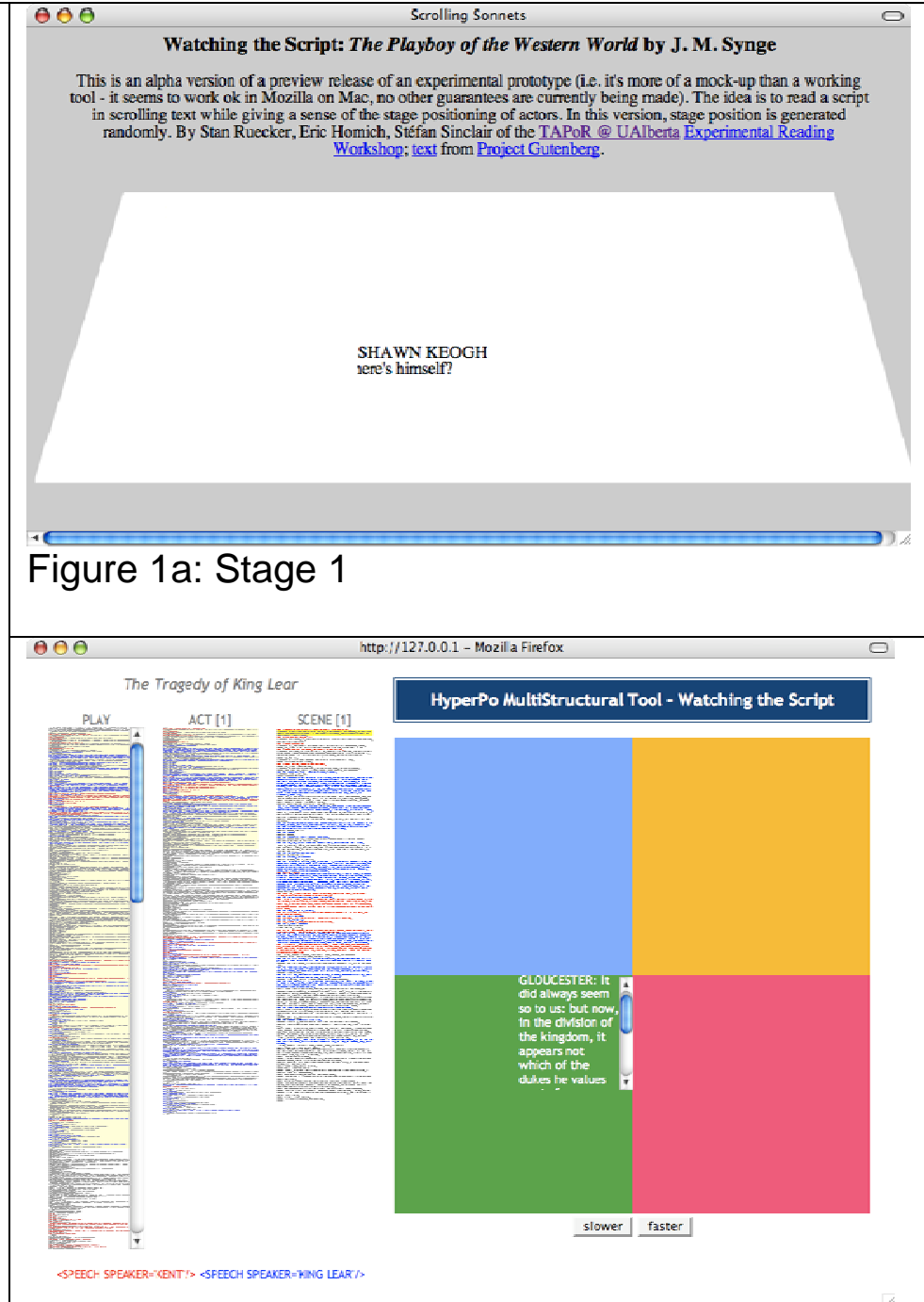

Figure 1b: Stage 2 


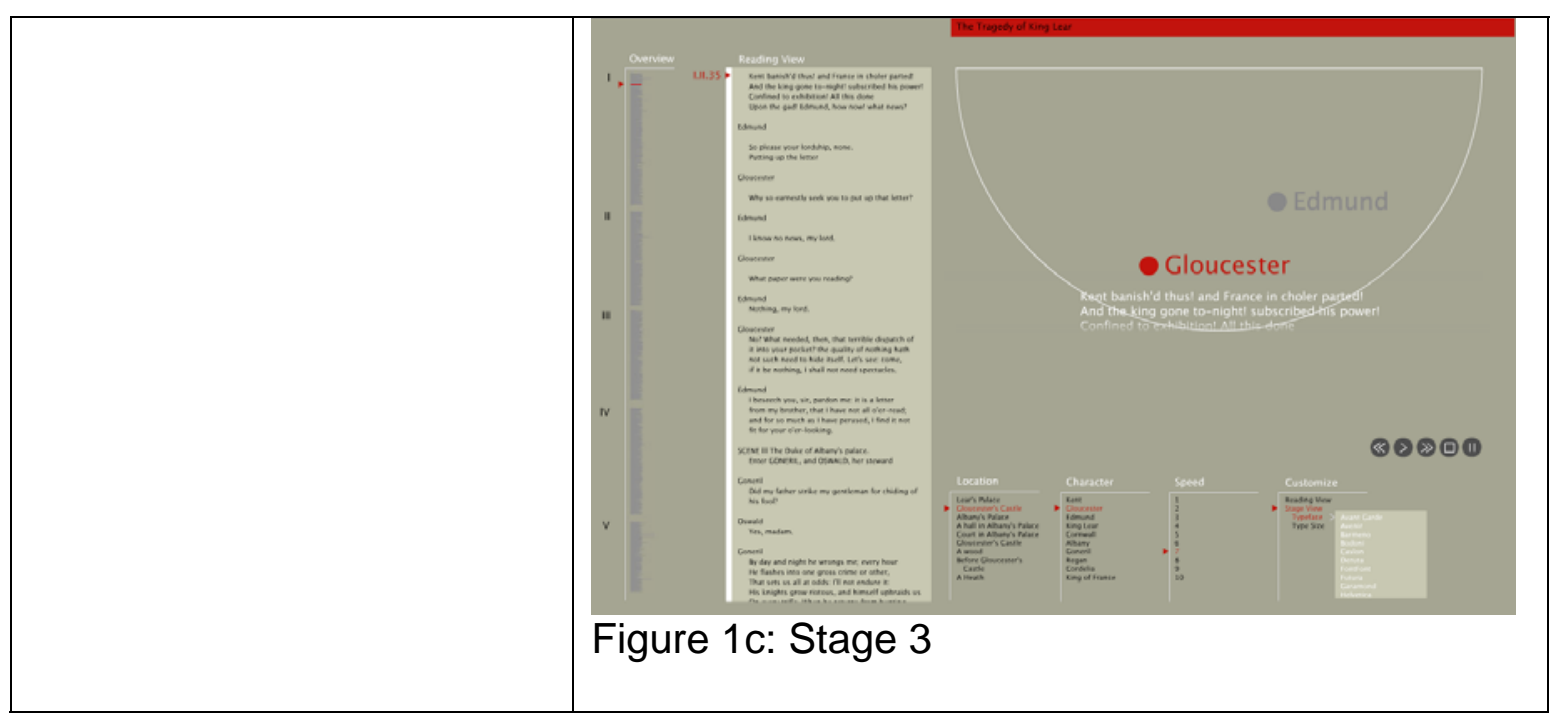

In Figure 1a, we see a bare minimum of design, with a skewed white rectangle representing a tilted stage. Characters and speeches are represented by default choices in typography.

Figure $1 \mathrm{~b}$ provides a marginal improvement to the design, with the addition of a colour and a stage marked into quadrants. Typographic details include microtext columns that have been colour-coded by character, and reverse text for character and speeches.

Figure $1 \mathrm{c}$ is a design that visually promises to reward investigation. Fonts for different purposes are clearly distinguished by size and colour. Character dots have been added to the stage to complement the text. The microtext and reading text are distinct columns that differ from each other in appearance. Tools are grouped along the bottom right, with headings and keylines to suggest that they are not reading texts but something else.

Attention to the details of the graphic design is in this case intrinsically related to the details of the functions of the system, such as the location and movement of characters and text. However, the list of additional qualities would not be complete without acknowledging that part of the attraction of the most recent iteration is the aesthetic function.

\section{Satisfaction}

Satisfaction can be derived from several different kinds of pleasurable experience, which may function individually or in harmony. Such pleasure can be understood as occurring at three levels: in the tool as an effective and attractive tool; in the high visual quality of the result of the process of using the tool; and in the fact that the project looks more polished when the findings are presented or published. 
In its extreme form, this value can result in forms of interface that are in some senses autotelic-they can become an end in themselves for some users, who find their attractions sufficient to make the system worth further attention, outside the context of any particular research task. For some members of the design community, the development of artifacts that can provide autotelic experiences is understood as one of the inevitable evolutionary consequences of a competitive marketplace (Jordan 2000), where tools that are merely functional are eventually replaced by tools that are functional and also easy to use; these tools in turn are replaced by those that are functional, usable, and also a source of pleasure.

\section{Clients}

There are three distinct groups that benefit directly from an increase in trust, confidence, and satisfaction - considering all three groups as forming an ecology is a key step in improving design practices in academic projects. The three groups are the designer, the user (or study participant), and the academic community. In discussing trust to this point, there has been the implicit assumption that the perceiver who is influenced by the aesthetic function is the study participant or the end user. The user may also possibly be a researcher, who is making use of the interface or visualization as a tool for studying other data, rather than as an object of study in its own right. However, there are also other people involved in the process, who are equally likely to respond in some way related to confidence. This group includes the academic community which receives and evaluates the work.

To begin with, there is the researcher or developer whose object of study is the tool. This person's confidence in the efficacy of the visualization system might be based on research results involving standard measures of performance and preference, or may simply be based on the conviction that the system is good at performing its function. However, in cases where the system is also manifestly well designed, with a recognizable attention to the aesthetic details, the developer may experience a satisfaction similar to that felt by the other users of the system.

Finally, there is the affective response of the academic community, who would experience the system not as users, but at one level removed, in the form of screen shots or other illustrations used in describing the visualization in the context of presenting research results. Figure 2 shows the cycle of production, use, and publication that ties these three communities together through the experience of the designed object. 


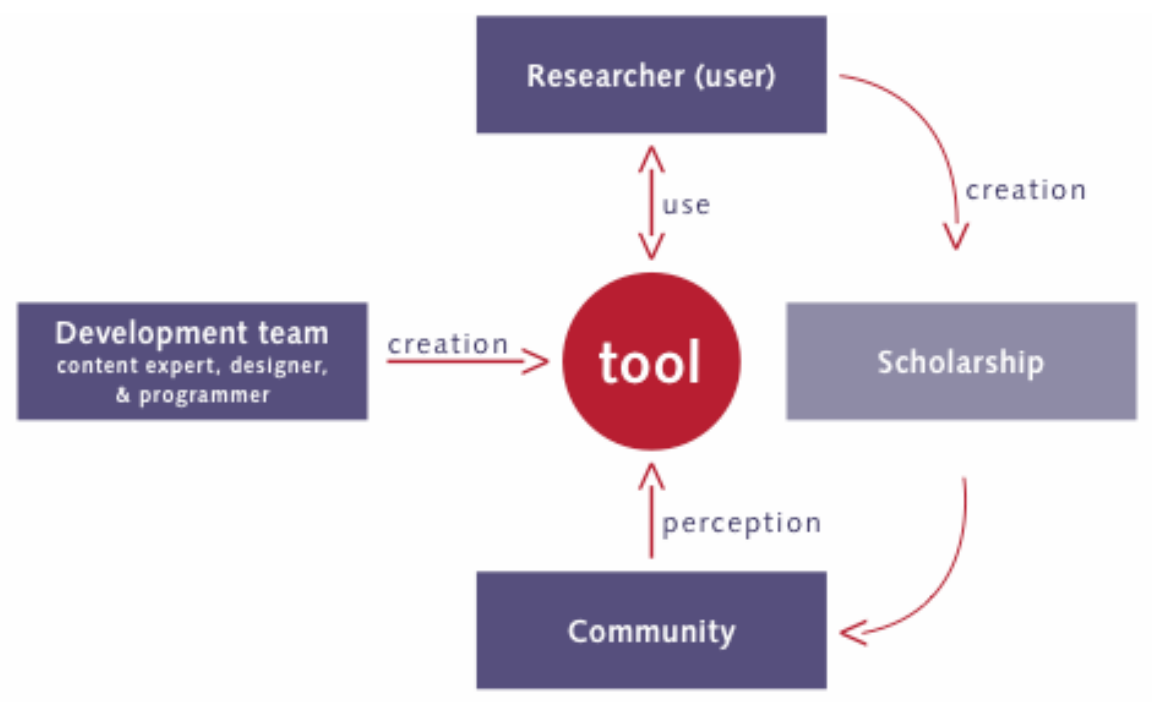

Figure 2. The developer creates a tool that the researcher uses to produce a result which is eventually published to the larger community. At each stage in the process, the perceptions of the developer, researcher, and reader can be influenced by the aesthetics of the designed object (Ruecker et al. 2005).

\section{Conclusions}

We have argued that a heightened attention to design is one strategy for increasing the trust of users and communities in academic research projects. One of our broader desired outcomes is to increase the profile of aesthetic visual design among researchers working in the digital humanities (a newer moniker for the 50 year-old discipline of humanities computing, which includes scholars in the humanities and information studies communities who make significant use of computers in their research and teaching). Increasing attention to design may involve gathering further information about the perceived role and significance of the aesthetic among this group. In addition, since the aesthetic impression begins within the first few milliseconds of perception, and consists in large part of the user's ability to identify the designer's attention to detail, there is a lack of well-defined measures that can be applied in evaluation of the aesthetic function. Explicit reporting by the user may have some meaning, but reactions at the tacit or unconscious level are not necessarily accessible for accurate reporting. Cognitive psychology may contribute to future work in this area through studies of implicit association, where subsequent behaviour can be shown to be influenced by earlier perceptions. That is, implicit associations relating to the aesthetic function might be indicated by secondary measures such as the willingness to engage in sustained use of the visualization even without a clear agenda, or through willingness to spend time troubleshooting. A related intriguing suggestion, by Larson and Picard (2005), is that one means of measuring aesthetic quality might be by relative subjective duration, where estimates of elapsed time made by participants who are enjoying themselves are significantly 
shorter than those made by participants who are not. The underlying suggestion, supported by previous studies, is that time flies while you are having fun. Further research is indicated.

\section{Works Cited}

Bertelsen, O. W. and S. Pold. "Criticism as an approach to interface aesthetics." Proceedings of NordiCHI'2004. Tampere: Finland, 2004. 23-32.

Bradley, J. and G. Rockwell. "What scientific visualization teaches us about text analysis. Consensus ex machina?" ALLC-ACH'94 abstracts. Paris. 1994. 203-204.

Carroll, N. Beyond Aesthetics: Philosophical Essays. Cambridge: Cambridge University Press, 2001.

Dillon, A. "Beyond usability: process, outcome and affect in human-computer interactions." Canadian Journal of Library and Information Science, 26(4) 2001. 57-69. Accessed 7 May 2007. http://www.ischool.utexas.edu/ adillon/publications.html

Dufrenne, M. The phenomenology of aesthetic experience, Northwestern University Press, Evanston, Illinois, 1973.

Fishwick, P.A., Ed. Aesthetic Computing. Cambridge: MIT Press, 2006. 
Fishwick, P.A., S. Diehl, J. Prophet, and J. Lowgren, J. "Perspectives in aesthetic computing." Leonardo. 38.2, 2005. 47-53.

Frascara, J. User-centred Graphic Design. London: Taylor and Francis, 1997.

Hallnas, L. and J. Redstrom "From use to presence. On the expressions and aesthetics of everyday computational things." $\underline{\text { ACM Transactions on }}$ Computer-Human Interaction (ToCHI). 9.2, 2002. 106-124.

Jennings, M. "Theory and Models for Creating Engaging and Immersive Ecommerce Websites." Proceedings of SIGCPR, Evanston, Illinois. NY: ACM Press, 2000. 77-85.

Jordan, P. Designing Pleasurable Products: An Introduction to the New Human Factors. London: Taylor and Francis, 2000.

Karvonen, K. "The beauty of simplicity." Proceedings of the ACM Conference on Universal Usability (CUU 2000). Washington DC, USA, November 16-17, 2000. 85-90.

Khaslavsky, J. and N. Shedroff, N. "Understanding the Seductive Experience." Communications of the ACM. 42.5, 1999. 45-49. 
Kurosu, M. and K. Kashimura. "Apparent Usability vs. Inherent Usability: Experimental analysis on the determinants of the apparent usability." Proceedings of CHI-95 Mosaic of Creativity. NY: ACM Press, 1995. 292293.

Larson, K. and R.W. Picard. "The Aesthetics of Reading." Paper presented at the Human-Computer Interaction Consortium, Colorado, February 2005. Accessed 7 May 2007. http://affect.media.mit.edu/pdfs/05.larsonpicard.pdf

Maeda, J. The Laws of Simplicity. Cambridge: MIT Press, 2006.

Munro, T. Toward Science in Aesthetics: Selected Essays. NY: Liberal Arts Press, 1956.

Ngo, D.C.L., L.S. Teo and J.G. Byrne. "Evaluating Interface Esthetics." Knowledge and Information Systems. 4, 2002. 46-79.

Petersen, M.G., O. Iverson, P. Krogh, and M. Ludvigsen. "Aesthetic interaction: a pragmatist's aesthetics of interactive systems." Proceedings of Designing Interactive Systems. NY: ACM Press, 2004. 269-276. 
Pujol, M. Design as a social practice. Public lecture. Trans. Jorge Frascara. Edmonton: Department of Art and Design, University of Alberta, 2001.

Pye, D. The nature and aesthetics of design. NY: Van Nostrand Reinhold, 1978.

Ruecker, S., S. Sinclair, and M. Radzikowska. "The Aesthetic Function: The role of visual communication design in interface research." Paper presented at the ACH/ALLC Conference 2005. Victoria, British Columbia: University of Victoria. June 15-18, 2005.

Ruecker, S., E. Homich, and S. Sinclair. "Watching the Script of Synge's Playboy of the Western World." Paper presented at $\mathrm{COCH} / \mathrm{COSH}$. Congress 2004. Winnipeg: University of Manitoba, 2004.

Ruecker, S., E. Homich and S. Sinclair. "Multi-level Document Visualization." Visible Language, 39.1, 2005. 33-42.

Ruecker S. "Experimental Interfaces Involving Visual Grouping During Browsing." Partnership: the Canadian Journal of Library and Information Practice and Research, 1,2006.

SSHRC. From Granting Council to Knowledge Council: Renewing the Social Sciences and Humanities in Canada. Ottawa: Social Sciences and 
Humanities Research Council of Canada, 2005. Accessed 7 May 2007. www.sshrc.ca/web/whatsnew/initiatives/transformation/reports/Volume3_fi nal_e.pdf

Sinclair. S., S. Gabriele, S. Ruecker and A. Sapp. "Digital Scripts on a Virtual Stage: The Design of New Online Tools for Drama Students." Proceedings of the Fifth IASTED International Conference, 2006. 155-159.

Tractinsky, N. "Aesthetics and apparent usability: empirically assessing cultural and methodological issues." Proceedings of CHI'97. NY: ACM Press, 1997. 115-122.

Tufte, E. The Cognitive Style of PowerPoint. Cheshire, CT: Graphics Press, 2003.

Udsen, L. E. and A. H. Jorgensen. "The aesthetic turn: unravelling recent aesthetic approaches to human-computer interaction." Digital Creativity. 16.4, 2005. 205-216. 